A new conjecture for this papyrus, especially for lines 9-15 which are the most difficult to interpret.

Este papiro contiene una carta privada, tal vez del siglo vp, de interpretación muy insegura. D. S. Crawford ${ }^{1}$ en la introducción a la misma dice: "The spelling and syntax, in keeping with the writing, are very far from correct, and this, combined with the uncertainty of the readings in a number of places and some lacunae, makes interpretation extremely difficult).

Tal vez el trozo que presenta mayor dificultad es el comprendido entre las líneas $9-15$. Como no parece que se haya propuesto ninguna interpretación a dichas líneas ${ }^{2}$, sugiero la presente por si puede merecer alguna consideración. Ante todo conviene transcribir dichas líneas según la editio princeps ${ }^{3}$ :

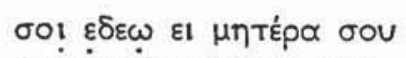

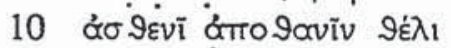

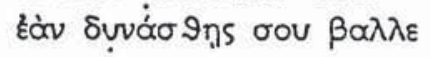

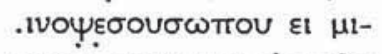

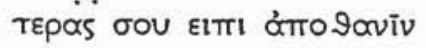

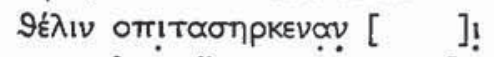

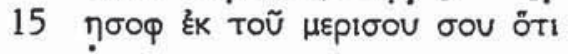

1 Por más que cite siempre a Crawford, debe tenerse presente lo que prologa E. G. Turner en abril de 1955: "On January 26th 1952 David Stewart Crawford and his wife fell victims to a riot in Cairo. The manuscript of this book, only just completed, escaped destruction". [...]. "Sir Harold Bell and myself have undertaken the responsability of seeing the work through the press?.

2 No consta en los volumenes de BL que mencionan la edición de PMichael.: IV, 1964; V, 1969.

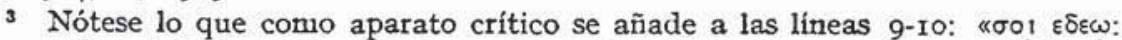

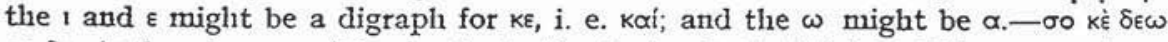
or $\delta \varepsilon \alpha$ is therefore possible. $\alpha$ rro Yaviv: the first $\alpha$ is doubtful, and there may have

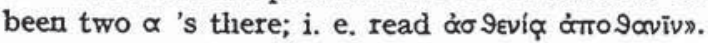


Línea 9: Atendiendo al $\sigma 01 \varepsilon \delta \varepsilon \omega$, vemos que Crawford dice sobre la segunda palabra: $« \varepsilon \delta \varepsilon \omega, \varepsilon \delta \varepsilon \alpha, \delta \varepsilon \omega$ or $\delta \varepsilon \alpha$ remains obscure. Could

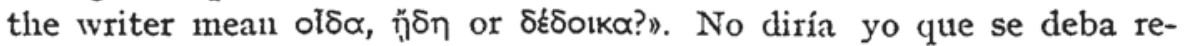
currir a esta interpretación, sino que tendría a $\varepsilon \delta \varepsilon \omega$ como una forma

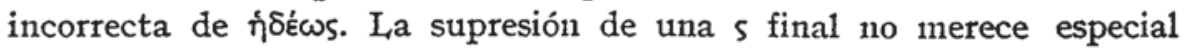
consideración. Así pues, dicho adverbio podría referirse a la frase an-

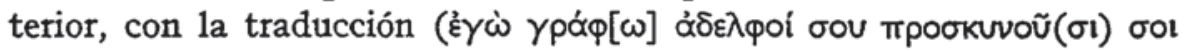
$\eta \dot{\gamma} \delta \xi \omega s)$ : "Te escribo que tus hermanos te saludan gustosamente». Sobre

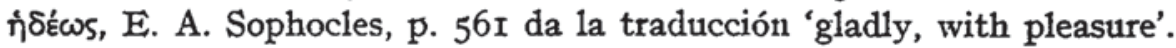

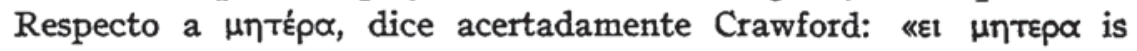

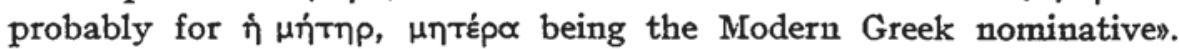
De mi parte preferiría tener a al no como artículo, sino como conjunción condicional. Sobre la omisión del artículo, puede recordarse 1o, que al hablar de los pronombres posesivos dice A. Thumb, Handbuch der neugrichischen Volkssprache, Strassburg 2 I9ro, p. 84: «Der bestimmite Artikel ist keinesweges unbedingt nötig».

Línea ro: Parece muy aceptable el comentario de la editio princeps:

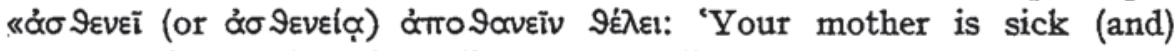
at the point of death', or 'is about to die of sickness'. Clearly $q^{\prime} \lambda_{\varepsilon t}$ must have its Modern Greek meaning of the immediate futuren.

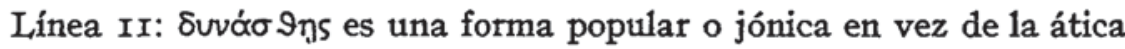

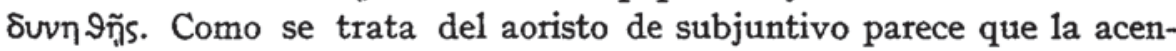
tuación debe ser $\delta u v \propto \sigma 9$ ñ̆s. Sobre la forma del aoristo, recuérdese la precisión de Mayser I r, p. Ig: "Die in Urkunden des III. Jahrh. auf-

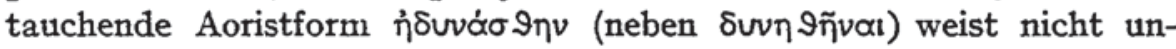
bedingt auf ionischen Ursprung hin: sie ist auch in der alten Dichtersprache ausserhalb Homers und bei Herodot und Xenophon belegt, kann also aus der Literatursprache in die kotvn übergangen sein».

Sobre la contextura de este período condicional, parece oportuno tener presente lo que indica B. G. Mandilaras, The Verb in the Greek Non-Literary Papyri, Athens 1973, p. 307: "The imperative is also used as apodosis of conditions expressing 'thought' (ठокєI), 'ability' ( $\delta \dot{v} v \alpha \mu \alpha 1)$ ), 'will' ( $(\dot{\hat{\prime}} \lambda \omega)$. In these constructions the imperative is under-

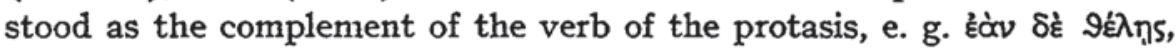

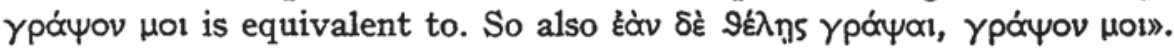

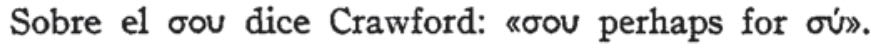

Lineas I2-I3: Anota Crawford: "The division of words at the beginning of line I2 is uncertain. Suggestions are $\sigma \omega \sigma \omega$ for $\sigma o v ́ \sigma \omega$, or 


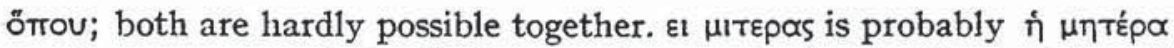
or unitnp again, and the final $\varsigma$ may be merely due to the doubling of the $\sigma$ of $\sigma o v$. $\varepsilon 1 \pi t$ presumably $\varepsilon i \pi \varepsilon v$ ».

Indiscutiblemente, la división de palabras al comienzo de la línea I2

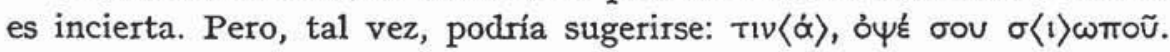
El $\mu \mathrm{t}-$.

Con respecto a la apócope de $\alpha$ en $\tau i v\langle\dot{\alpha}\rangle$, puede aducirse un caso

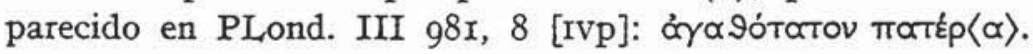

ó $\psi \varepsilon ́$ en su obvia significación adverbial se traduce por "spät» (Preisigke, Wörterbuch II, 2I6). Pero conviene no olvidar que también puede desempeñar una función preposicional, como lo indica W. Bauer, Wörterbuch, Berlín 5 I958, p. IIg2: «als (uneigtl.) Präp. mit d. Gen. gebr.

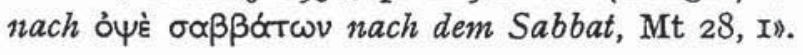

$\sigma\langle\mathrm{t}\rangle \omega \pi$ Toṽ: como fácilmente se advierte en la integrada raíz de la palabra ( $\sigma \iota \omega^{\prime} \alpha \dot{\omega}, \sigma \iota \omega \pi \eta \dot{)}$, se incluye en dicho término la idea de 'silen-

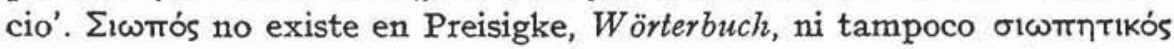

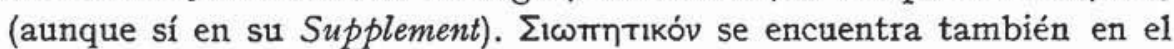
Supplement del LSJ, con el significado (p. I33): 'novice's fee in a mystery cult'. Dicha palabra está registrada en PMich. VIII 5II, 3.6 [mip]. Con respecto a la línea 3 se anota en la editio princeps del indicado PMich.:

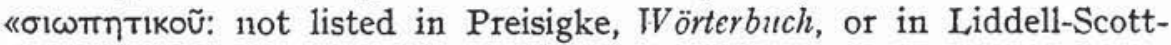
Jones. The eighth edition of Liddell-Scott records the adjective but restricts its comment to 'taciturn, Byz.'. Only four references from late authors (4th-6th cent.) are cited in Sophocles' Lexicon and Stephanus' Thesaurus». De todo lo dicho parece que $\sigma\langle 1\rangle \omega \pi$ ós o incluso $\sigma\langle\imath\rangle-$ $\omega \pi\langle\eta \tau ı K\rangle$ ós puede entenderse como 'taciturno, reservado'. Así, pues,

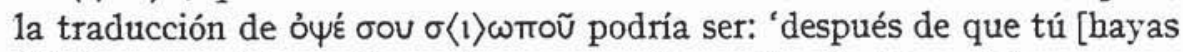
sido] reservado', es decir, 'con suma discreción de tu parte'.

No se me escapa que tomando a ó $\psi \varepsilon$ como adverbio, podría también traducirse $\delta \psi \xi \dot{\varepsilon}, \sigma \circ \tilde{~} \sigma\langle 1\rangle \omega \pi$ Tõ por 'al atardecer y discretamente'.

Linea I4: En la correspondiente nota leemos: "The division of the words after $9 \dot{\varepsilon} \lambda ı$ is uncertain». Tal vez se pueda proponer: ótrítas

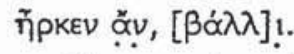

En cuanto a ómít $\alpha$ s, puede quizá tratarse de ómótav. La confusión de $-v$ y $-s$ finales no ofrece dificultad. Recuérdese lo que ya decía Fr. Völker, Papyrorum Graecarum syntaxis specimen, Bonnae Igoo, p. 36 : "Cumque et $-v$ et $-s$ in fine vocabulorum spiritu tenuissimo pronuntiarentur aut omnino mutae fierent, non est mirum quod nonnunquam inter se mixtae videntun. 


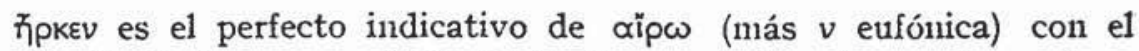
significado de 'wegnegmen' (Preisigke I, p. 35), 'lominem: ab uno loco in alium amoueo' ('Zorell, p. 39), 'tolgo di mezzo' (Rocci, p. 44). Eu cuanto a la intromisión del perfecto en la esfera del aoristo por lo que respecta al significado, cf. L. Radermacher, Neutestanentliche Grammatik. Das Griechisch des Neuen Testaments im Zusammenhang mit der Volkssprache, Tübingen ${ }_{2}$ I925, p. I5O.

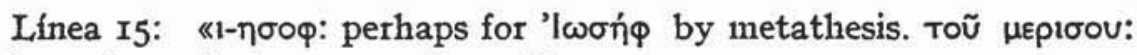

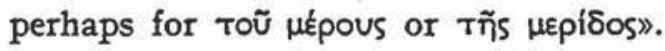

Antes de ofrecer la nueva transcripción de las líneas 9-15, conforme a lo que hasta aqui se ha indicado, es conveniente copiar lo que sigue

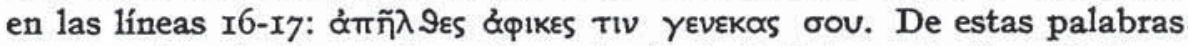
propone Crawford, en primer lugar, la siguiente interpretación: "Perhaps

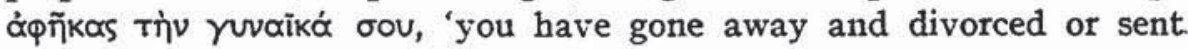
away your wife'n.

Esto supuesto, veamos la nueva sugerencia de lectura:

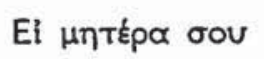

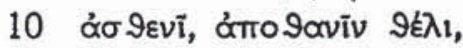

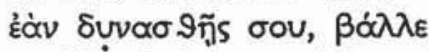

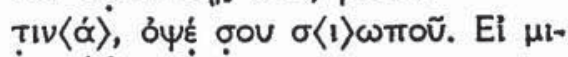

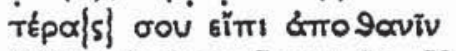

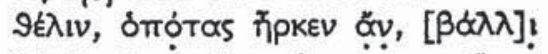

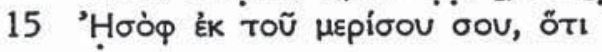

La correspondiente traducción con la interpretación de las líneas i6I7, dada por Crawford, es la siguiente:

"Si tu madre enferma de gravedad, con suma discreción de tu parte envía, si puedes, a alguien. [Pero] si tu madre, [consciente de su estado], llega a decir que está a punto de morir, entonces, caso que hubiera rechazado (quitado de enmedio) [al otro], manda, de tu distrito a José(?), [pues se encuentra sola], ya que tú te has marchado y has repudiado a tu mujen.

\section{José O'CALLAGHan}

\section{Pontificio Instituto Bíblico}

Roma 